\title{
Multiobjective $H_{2} / H_{\infty}$ Control of a Pitch Regulated Wind Turbine for Mechanical Load Reduction
}

\author{
Fabien Lescher ${ }^{* \ddagger}$, Jing Yun Zhao*, André Martinez* \\ * ERPA-EIGSI \\ 26 rue Vaux Le Foletier, 17041 La Rochelle \\ ¥ LAGIS-Ecole Centrale de Lille \\ Cité Scientifique - BP 4859651 Villeneuve d'Ascq Cedex \\ E-mail: fabien.lescher@eigsi.fr
}

\begin{abstract}
The paper deals with the design of a control system for a variable-speed pitch-regulated wind turbine in the operating region corresponding to wind speeds above rated wind speed. The control objectives are mostly to regulate the plant output power and to reduce the mechanical fatigue of the plant components. The controller is designed from a non linear model of the system which takes into account the blades, shaft and tower flexibilities. A Linear Matrix Inequalities (LMI) formulation of the control problem permits to obtain a Linear Parameter Varying (LPV) controller optimizing, through $\mathrm{H}_{2} / H_{\infty}$ norm minimization, a criteria close to mechanical fatigue affecting the plant. The proposed controller performances are then compared in simulation with those of a gain scheduling Linear Quadratic Gaussian (LQG) controller and with a gain scheduling PI controller, and is seen to be more efficient.
\end{abstract}

Keywords: Wind turbine, control, LMI optimization, mechanical fatigue.

\section{Introduction}

Wind energy has widely grown during the last decades and nowadays is the most competitive form of renewable energy. Nonetheless, wind energy is not yet cost effective. In consequence, the development of new technology will be crucial that the wind energy penetrates into electricity market successfully. Implementation of advanced control systems is considered as a promising way to improve wind turbine conversion system and to decrease wind energy cost [1]. Wind turbine control objectives are mainly to optimize wind energy conversion, and to reduce dynamic loads experienced by the mechanical structure of the plant [2]. Indeed, dynamic loads hardly affect the lifetime of wind turbines and mainly determine mechanic components design [3]. Most damaging stresses affecting the mechanical structure of the wind turbine occur for high wind speeds, for which the controller task consists in regulating produced power at the nominal power of the generator, acting on both the pitch angle of the blades and the electromagnetic torque of the generator.

Most of papers dealing with the design of controllers for wind turbines takes interest only in alleviating loads experienced by the drive train, whereas this component is far from being the most expensive.Moreover, it has been noticed that the pitch action has good ability for reducing the very few damped vibrations of the blades and the tower [4]. Besides, the usual control structures employed for the alleviation of the loads affecting the whole plant are composed by several separate and decoupled loops, each loop being tuned for one objective, whereas the considered control problem is a multivariable and multiobjective one [4][5]. In this paper, a multivariable controller is presented for this purpose. By taking into account all control objectives and all model properties for the design of the multivariable controller, an improvement of the performances of the controller can be expected. The presented controller is designed from a mathematical model of the system describing main plant 
flexibilities.

The selected controller synthesis method is based on a mixed $H_{2} / H_{\infty}$ norm optimization of the transfer functions between performances and wind speed variations. This method is based on a Linear Matrix Inequalities (LMI) formulation of the control problem, and allows to partially vanish the resonance peaks caused by the flexibility of the components, and which are mainly responsible of the mechanical fatigue of the plant. Moreover the proposed controller takes into account the non linear behavior of the plant, notably due to the aerodynamic part, by adapting its parameters to operating conditions. This gain scheduling controller is designed from a non linear description of the aerodynamic properties of the blades, by transforming the model of the system into a Linear Parameter Varying (LPV) model.

\section{System Description}

The structure of a variable speed, pitch regulated wind energy conversion system is presented in Figure 1. A model of the mechanical structure of a wind turbine has firstly been established using Lagrange's equations to take into account the mechanical dynamics of the structure, and has been coupled with a non linear model of the aerodynamic properties of the blades. The system is formed by the wind turbine, the drive train, and by a generator unit, composed by the generator and the static converter connected to the electrical grid. The control system acts on generator in order to apply the reference electromagnetic torque $T_{G \text {,ref }}$ and on the pitch actuator in order to control the pitch angle of the blades $\beta$, calculated from the measurements of the rotational speed of the shaft at the generator side, and of the speed of the tower by an accelerometer located at the top of the tower.

The aerodynamics conversion process of the turbine rotor is characterized by the extracted torque $T_{a e r o}$ and by the out-of-plane thrust force $F_{\text {aero }}$, which are functions of the air mass density $\rho$, the wind velocity $v$, the rotational speed of the turbine $\omega_{T}$, the horizontal blades speed $\dot{x}_{P}$ and the power and

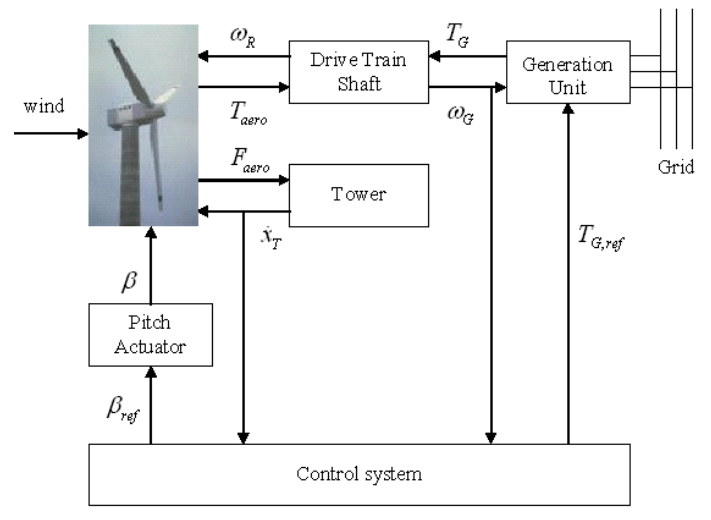

Figure 1: Wind energy conversion system structure.

thrust coefficients $C_{p}$ and $C_{t}$ :

$$
\begin{aligned}
T_{\text {aero }} & =\frac{1}{2} \rho \pi R^{2} \frac{\left(v-\dot{x}_{P}\right)^{3}}{\omega_{T}} C_{p} \\
F_{\text {aero }} & =\frac{1}{2} \rho \pi R^{2}\left(v-\dot{x}_{P}\right)^{2} C_{t}
\end{aligned}
$$

with $R$ the length of the rotor blades. The aerodynamic coefficients $C_{p}$ and $C_{t}$ are non linear functions depending on blades pitch angle $\beta$ and tip speed ratio $\lambda$ defined by the relation:

$$
\lambda=\frac{\omega_{T} R}{v} .
$$

In addition to the plant model, a wind model is established for the control design model. Wind velocity in a fixed point of space has known properties in the frequency range, represented by the Van der Hoven spectrum (Figure 2). The turbulent component of the wind speed is modeled as a first order dynamic process disturbed by a Gaussian white noise $m_{v}(t)$, with a time constant $T_{v}$ calculated in order to take into account the stochastic properties of the wind speed [7]:

$$
\dot{v}_{t}=-\frac{1}{T_{v}} v_{t}(t)+m_{v}(t)
$$

Moreover, wind shear and tower shadow effects are modeled by adding to the wind speed a periodic signal of frequency corresponding to three times the frequency of revolution of the turbine $(3-P$ rotational effects). 


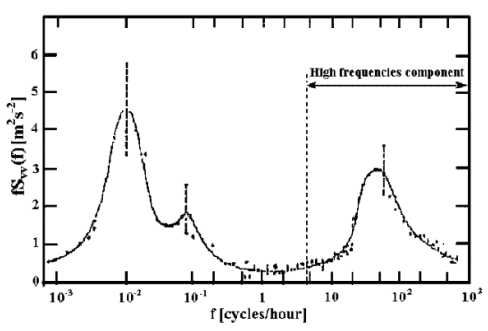

Figure 2: Spectrum of horizontal wind speed

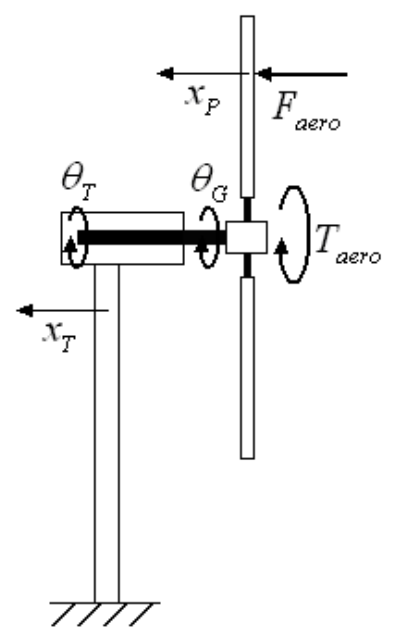

Figure 3: Wind Turbine Mechanical Structure

The mechanical model describing the structure of the plant has three degrees of freedom: the flexion of the blades (blades flap motion) and of the tower (tower fore-aft motion) in the direction of the wind, and the torsion of the drive train shaft (Figure 3). A spring-damper system representation is used to describe the flexibility of each component. Moreover, the three blades are supposed to move conjointly and to be affected by the same forces at the same time. A linear model of this structure is established using Lagrange's equations and is coupled with the non linear aerodynamic model.

The electrical subsystem, corresponding to the generation unit, composed by the generator and the power electronic components, has very fast dynamics compared with dynamics of the other subsystems. Consequently, and considering the objectives of the study, the electrical dynamics are neglected. Hence, electromagnetic torque $T_{G}$ is sup- posed equal to its reference $T_{G \text {,ref }}$.

The pitch actuator subsystem represents the hydraulic or electric system which makes the blades revolve around their lengthwise axis. This system is described by a first order transfer function with a time constant $T_{\beta}$. Physical limitations on pitch rate and pitch amplitude are also taken into account for controller synthesis and simulations.

\section{Controller synthesis}

\subsection{Control task}

In the studied operating zone, which corresponds to wind speeds above rated wind speed, the system has to operate at a fix rotational speed, the nominal speed of the generator, and has to produce a fix electric power, the nominal power of generator. Another controller objective is to limit the mechanical fatigue of the components, especially blades, shaft and tower. These two objectives are clearly contradictory, and therefore a trade off between these two objectives has to be optimized.

Whereas the power regulation objective can easily be expressed with the parameters which describe the system operation, it is practically impossible to express precisely the mechanical fatigue of components with these parameters. Indeed, mechanical fatigue is usually estimated from time series of mechanical loads experienced by components by applying the Rainflow Counting Algorithm and Miner's rules, in order to take into account both amplitude and number of occurrences of the loads variations [8]. Then, the reduction of the mechanical fatigue cannot be explicitly taken into account during controller synthesis. Nevertheless, such frequency domain based criteria as $H_{2}$ or $H_{\infty}$ can be very useful to express performances similar to mechanical fatigue. Actually, these synthesis techniques allow to shape transfer functions of channels between performances and external disturbances. Hence, a criteria representing both amplitude and frequency of loads affecting components can be expressed and optimized by these synthesis technique. This criteria can then be brought closer to the mechanical fatigue. 


\subsection{Controller synthesis}

The control system is designed from a Linear Parameter Varying (LPV) representation of the wind turbine: the model of the system is firstly linearized along the reference trajectory, corresponding to nominal speed and nominal produced power operation, and is put into a state space representation, with state matrices depending on the operating point on the reference trajectory. This LPV model permits to consider the non linearities in the expressions of the aerodynamic torque $T_{\text {aero }}$ and thrust force $F_{\text {aero }}$ (Eq.1). For this purpose, the aerodynamic coefficients describing the dependence of $T_{\text {aero }}$ and $F_{\text {aero }}$ with state vector components, $\frac{\partial T_{a e r o}}{\partial \omega_{T}}, \frac{\partial T_{a e r o}}{\partial v}, \frac{\partial T_{a e r o}}{\partial \beta}$, and $\frac{\partial F_{a e r o}}{\partial \omega_{T}}, \frac{\partial F_{a e r o}}{\partial v}, \frac{\partial F_{a e r o}}{\partial \beta}$ are approximated by affine functions of the square of pitch angle $\beta^{2}$. Thus, the obtained LPV state space representation of the wind turbine behavior along the reference trajectory is:

$$
\begin{aligned}
\dot{x} & =\left(A_{0}+\beta^{2} A_{1}\right) x+B u+G w \\
y & =C_{y} x \\
z & =C_{z} x+D u
\end{aligned}
$$

with the state vector $x^{T}=$ $\left(\begin{array}{lllllllll}\theta_{T}-\theta_{G} & x_{B} & x_{T} & \omega_{T} & \omega_{G} & \dot{x_{B}} & \dot{x_{T}} & \beta & v_{t}\end{array}\right)$,

the controlled input vector $u=\left(\begin{array}{cc}T_{g} & \beta_{\text {ref }}\end{array}\right)^{T}$, the external disturbance $w=m_{v}$, the measured output vector $y=\left(\begin{array}{lll}\omega_{G} & \dot{x_{T}}\end{array}\right)^{T}$. The vector $z$ represents the performances outputs, and is composed by the produced power $P_{\text {elec }}$, the drive train torsion torque $T_{D}$ and the blades and tower flexion forces $F_{B}$ and $F_{T}$, expressed by:

$$
\begin{aligned}
& T_{D}=d_{D}\left(\omega_{T}-\omega_{G}\right)+k_{D}\left(\theta_{T}-\theta_{G}\right) \\
& F_{B}=d_{B} \dot{x_{B}}+k_{B} x_{B} \\
& F_{T}=d_{T} \dot{x_{T}}+k_{B} x_{T}
\end{aligned}
$$

with $d$ and $k$ the damper and spring coefficients of the corresponding components.

The selected structure for the controller is a dynamic output feedback whose parameters are also dependant on the parameter $\beta^{2}$ :

$$
\begin{aligned}
\dot{x}_{K} & =\left(A_{K}\left(\beta^{2}\right) x_{K}+B_{K}\left(\beta^{2}\right) y\right. \\
u & =C_{K}\left(\beta^{2}\right) x_{K}+D_{K}\left(\beta^{2}\right) y
\end{aligned}
$$

The controller objectives are firstly to ensure stability along the reference trajectory, in spite of the variations of the system parameters, and to ensure good performances of the selected outputs, by minimizing the $\mathrm{H}_{2}$ norm of the variations of the produced power, and by guaranteeing an $H_{\infty}$ norm performance of the shaft torsion torque and blades and tower flexion forces. $H_{2}$ norm minimization permits to reduce produced power variations for the whole frequency range, whereas $H_{\infty}$ cost represents the maximum over the frequency range of the bode response of the outputs to a variation of wind speed.

Moreover, in order to consider the properties of the control objectives in the frequency range, the system (5) is augmented with weighting functions as shown in the block diagram in Fig 4. The weighting function $W_{P_{\text {elec }}}$ is a low pass filter that tends to ensure to the produced power a good tracking at low frequency. At high frequency, a good tracking of $P_{\text {elec }}$ is not reached because it would induce an increase of dynamical loads on the drive train and of pitch actuation. $W_{\beta_{\text {ref }}}$ is a high pass filter and permits to prevent pitch demand from being too important at high frequency. The high pass filters $W_{T_{D}}, W_{F_{T}}$ and $W_{F_{P}}$ ensure weak variations of the drive train torque, blades and tower forces for the frequencies in amount of the resonance frequency, and therefore a reduction of the plant mechanical fatigue.

The control problem can then be formulated as

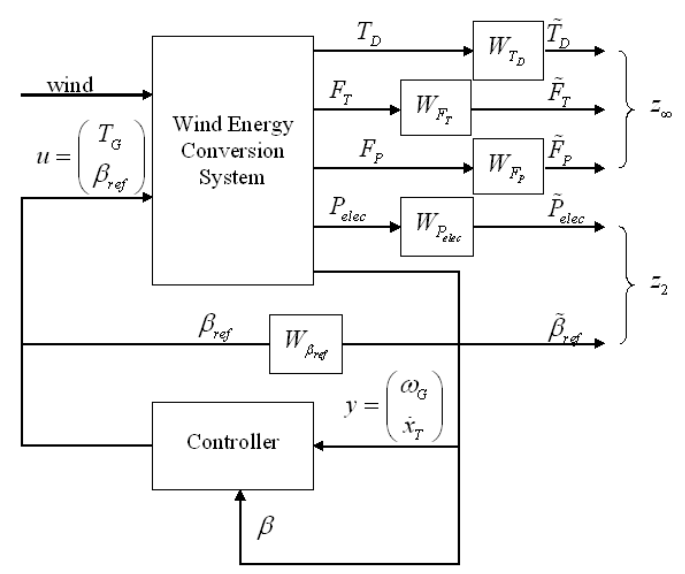

Figure 4: Closed Loop System Structure 
the following optimization problem:

$$
\begin{aligned}
\min \nu \text { such as: } & \\
\left\|\tilde{z}_{\infty}\right\|_{\infty} & <\gamma\left\|\tilde{z}_{\infty}\right\|_{\infty_{o . l}} \\
\left\|\tilde{z}_{2}\right\|_{2} & <\nu
\end{aligned}
$$

with $\left\|\tilde{z}_{\infty}\right\|_{\infty_{0, l}}$ the $H_{\infty}$ cost of $\tilde{z}_{\infty}$ for the open-loop system. The coefficient $\gamma$ has to be selected to ensure a good trade off between the two contradictory objectives.

An additional constraint is to enforce the poles of the closed loop system to be located in a circle around the origin in order to provide the system from having too fast dynamics.

The control problem (7) of the LPV system (5) can be formulated as a convex optimization problem with Linear Matrix Inequalities, and can then be solved by available softwares [6].

\section{Simulation Results}

The proposed controller is compared, at the sight of simulation results, with two other existing controllers, a gain scheduling PI-based controller, and a multivariable gain scheduling LQG one. The simulations are based on a dynamic model implemented in Matlab-Simulink containing:

- non linear and stationary aerodynamics, with $3-P$ rotational effects.

- flexible drive train model, flexible tower model including first fore-aft mode, flexible blades in flapwise direction,

- pitch actuator limitations on pitch rate $\left( \pm 10^{\circ} / \mathrm{s}\right)$ and pitch amplitude.

The simulated wind speed respects stochastic properties of Van der Hoven spectra with high fluctuations.

As mentioned in [4] and [5], the PI-based controller is designed by calculating controllers for two separate loops: firstly, a PI controller is tuned to guarantee power regulation from generator speed measurement. Then a tower speed feedback is designed to increase tower fore aft damping, by calculating a controller which appropriately filters blades flap excitation and which does not interact with the bandwidth of the first loop. Unlike the proposed controller, this controller acts only on pitch angle.

\begin{tabular}{|l||c|c|c|}
\hline Controller & Shaft & Tower & Blades \\
\hline LPV controller & $63.0 \%$ & $90.1 \%$ & $75.8 \%$ \\
\hline LQG controller & $139.8 \%$ & $105.3 \%$ & $96.3 \%$ \\
\hline PI controller & $100 \%$ & $100 \%$ & $100 \%$ \\
\hline
\end{tabular}

Table 1: Equivalent Load: Ratio between the different controllers and PI controller.

The gain scheduling LQG controller is designed from the same augmented model and with the same performances outputs as the proposed controller. The LQG methodology permits to design a multivariable controller which optimizes a time domain quadratic criteria representing a trade off between the different control objectives. Unlike the proposed LPV design methodology, the LQG design does not provide guarantees of stability ad performances along the reference trajectory, but only in several operating points on this trajectory [9]. Actually, one LQG controller has to be designed at each linearization point of the trajectory, and a gain scheduling process has to be used to interpolate the different LQG controllers. Moreover, and unlike the proposed method, the LQG design does not provide any specification of performance in the frequency range such as $H_{\infty}$ or $H_{2}$ norms.

The controllers performances are compared for both power regulation and alleviation of mechanical fatigue. Evaluation of mechanical fatigue is provided by using the Rainflow Counting Algorithm, which calculates the number of load cycles from time domain simulations results, and the fatigue equivalent load for each component.

Temporal series of wind speed, produced power $P_{\text {elec }}$ and pitch angle $\beta$ are presented in Figure 5. Fatigue equivalent loads for the shaft, blades and tower are calculated from the simulations, and Table 1 presents the ratio between the equivalent loads obtained with the different controllers and with the PI-controller.

With the same pitch actuation level for the three controllers, the proposed LPV controller is seen to be more effective for the two selected control objectives, especially for for mechanical fatigue reduction for each component. A sensitive gain of lifetime of the mechanical components of the plant, or a sensitive reduction of the mass, and consequently of the cost of these components can then be expected. 

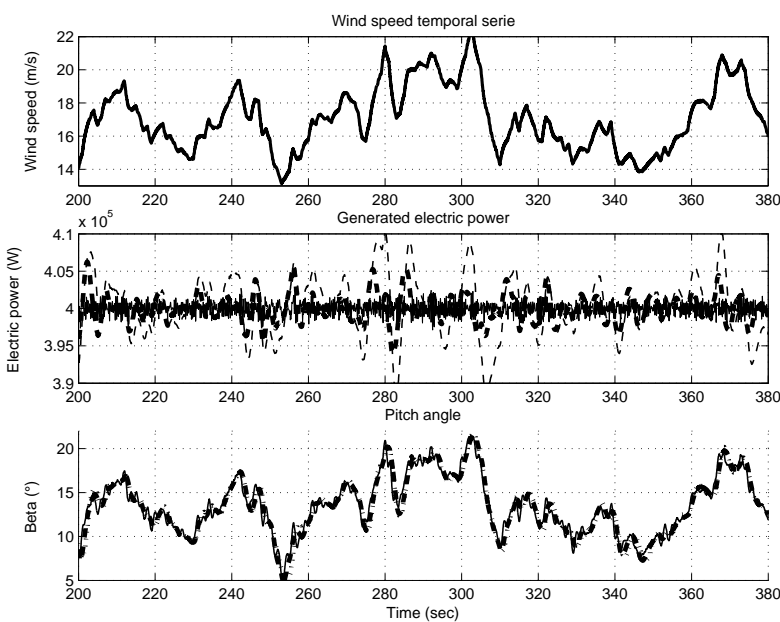

Figure 5: Temporal series: full: LPV controller, thin dash: PI-controller, thick dash: LQG controller.

\section{Conclusion}

In this paper, a non linear, multiobjective and multivariable controller has been presented and has been seen able to improve sensitively, at the sight of simulation results, the plant performances. Indeed, a frequency domain criteria depending on the internal forces affecting the plant structure, close to the mechanical fatigue criteria, is expressed and is minimized by the selected control design methodology. This controller provides then a good alleviation of mechanical loads, and by the way a reduction of the mechanical fatigue and then a reduction of the cost of the mechanical structure of the plant, or an increase of the lifetime.

\section{References}

[1] A.D. Şahin, Progress and recent trends in wind energy, Progress in Energy and Combustion Science, vol.30, 2004, pp.501-543.

[2] W.E. Leithead and B. Connor, "Control of variable speed wind turbines: design task", Int. Journal of Control, vol.13, 2000, pp.11891212.

[3] M.J. Balas, A. Wright, M.M. Hand, K. Stol, Dynamics and control of horizontal axis wind turbines, Proceedings of the American Control conference, Denver, Colorado, June, 2003.

[4] E.A. Bossanyi, The design of Closed Loop Controllers for Wind Turbines, Wind Energy 2000, vol.3, 2001, pp.149-163.

[5] W.E. Leithead, S. Dominguez and C.J. Spruce, Analysis of Tower/Blade interaction in the cancellation of the tower fore-aft mode via control, EWEC 04, London, UK, 2004.

[6] P. Apkarian and R.J. Adams, Advanced GainScheduling Techniques for Uncertain Systems, IEEE Transactions on Control Systems Technology, Vol.6, no.1, pp.213-32, 1998.

[7] C. Nichita, D. Luca, B. Dakyo and E. Ceanga, Large band simulation of the wind speed for real time wind turbine simulators, IEEE Transactions on Energy Conversion, vol.17, pp.523-529, 2002.

[8] M. Matsuiski and T.Endo, Fatigue of metals subjected to varying stress, Japan Soc. Mech. Engrg, 1969.

[9] F. Lescher, J.Y. Zhao and A. Martinez, LQG Multiple Model Control of a Variable Speed, Pitch regulated Wind Turbine, 17th IMACS World Congress, Paris, France, 2005. 